\title{
Do Material Discontinuities in Silica Affect Vibration
}

\section{Modes?}

Victor V. Volkov, David J. Belton, Carole C. Perry*

Interdisciplinary Biomedical Research Centre, School of Science and Technology, Nottingham Trent University, Clifton Lane, Nottingham NG11 8NS, United Kingdom.

\begin{abstract}
Structural properties of bioinorganic composites are of current interest in the areas of drug delivery, bone repair and biomimetics. In such composite systems, structural analysis is enhanced when we combine methods of spectroscopy and simulation. Depending on size and shape, structural discontinuities of inorganic matter may modulate the optical response of a bound molecule. Using Density Functional Theory we explore the effects of a local field next to the surface of a silica cluster on frequencies of methyl stretching modes of associated methanols. Computation results predict that the electrostatic potential modulated by structural discontinuities of silica should not contribute to any systematic frequency shifts for normal modes of a guest molecule. Regardless of position, the methyl stretching modes of methanol demonstrate sensitivity only to the local chemistry of bonding with silanols, which may lead either to a low or to high frequency shift for vibrations. In support, experimental studies of deuterated methanol at impurity levels in water show uniform broadening of resonances of Carbon-Deuterium stretching modes in
\end{abstract}


the presence of both crystalline and amorphous silica nanoparticles. The significance of these findings is that the spectral responses of guest molecules on such surfaces should not be subject to bias introduced by edge effects.

\section{INTRODUCTION}

The mass of Earth's crust is 59 percent silica. ${ }^{1}$ It exists in many crystalline forms as well as an amorphous mineral, ${ }^{2}$ and the latter form, due to its role in engineering ${ }^{3,4}$ and in the pharmaceutical industry, ${ }^{5}$ is one of the best-studied amorphous materials. ${ }^{6,7}$ However, because engineering of inorganic interfaces is at the center of many technological advances where both miniaturization and accuracy in control of chemical composition play key roles in achieving major breakthroughs, ${ }^{8}$

the structural and electronic properties of silica remain a focus of contemporary research. ${ }^{9}$ Achieving new and enhanced properties for novel materials is based on our capacity to predict how size may affect the physicochemical responses of the designed engineered nanomaterials. The challenge is to bridge between experiment and theory when engineered matter is on the size scale of a micron or below. For clusters of small sizes, the role of the surface increases, and the constraint of crystalline periodicity may become relaxed. In this respect, confirming structural and electronic properties as observed in experiment become an important task for theory to predict beyond what is known and to offer novel opportunities.

Contemporary inorganic synthesis and engineering require accurate prediction of the properties of matter where the number of atoms is relatively large. ${ }^{10}$ In this respect, we can either conceptualize a relatively small subset of structural moieties to explore using tools of quantum chemistry or we have to think how to anticipate possible inadequacies of theoretical predictions from classical simulations of large systems with many degrees of freedom. Both approaches find 
active development in contemporary research in application to silica and silica related materials. ${ }^{9}$ For example, using ab initio molecular dynamics simulations with plane waves under periodic boundary conditions ${ }^{11-13}$ and gaussian wave-functions with Density Functional Theory (DFT), ${ }^{14,15}$ a recent series of publications addresses the electronic properties of silica and absorption of water and formic acid to its surface in dependence on chemical defects, disorder and structural variance at the quantum level. Furthermore, being transparent to visible light and relatively inert in bio- and photo-physical processes, amorphous silica is a valuable substrate in the pharmaceutical industry to assist transport of associated molecules. This behavior stimulates structural studies of guest molecules next to silica interfaces. For instance, association of pyridine derivatives ${ }^{16,17}$ and adsorption of neutral glycine ${ }^{18}$ to silica interfaces has been explored by DFT simulations of structural composites of these molecules with hydroxylated silica $\mathrm{SiH}_{3} \mathrm{OH}$ and/or $\mathrm{H}_{2} \mathrm{SiOSiH}_{2}(\mathrm{OH})$ structural motifs. In recent studies, the role of water in interaction of aspirin ${ }^{19}$ and ibuprofen with an amorphous silica surface ${ }^{20}$ has been explored by means of DFT simulations using pseudoatomic orbitals and a specifically attuned composite approach, respectively. Furthermore, employing structural hierarchy, recently, protective encapsulation of a fluorophore in silica has been predicted and described using the TD-B3LYP/N07D level of theory within the ONIOM(QM:MM) scheme ${ }^{21}$ along with research on fluorophores, ${ }^{22}$ where a fluorophore was manually docked onto the surface in such a way that the two oxygen atoms of the carboxylate group strongly interact with one isolated silanol group of a $15 \AA$ thick slab $\left(\mathrm{Si}_{60} \mathrm{O}_{134} \mathrm{H}_{28}\right)$ extracted form an amorphous silica bulk. ${ }^{23}$

As we have mentioned, classical molecular dynamics (MD) simulation is the theoretical alternative when we wish to address properties of very large molecular systems and bio-inorganic composites. This is particularly valuable in our studies when we target characterization of binding 
to silica of relatively large polypeptides. ${ }^{10,24,25}$ Often, structural characterizations in such relatively large, heterogeneous and disordered systems may critically depend on interpretations of Infrared and Raman responses. To accomplish this with some degree of rigour, one would have to bring from MD simulation a relevant structural extract into the quantum environment to perform a higher quality optimization and normal mode analysis. In this respect, it is important to ask the question - if extraction of a truncation of a silicon oxide segment with a guest molecule next to it would create spurious fields at the edges that would systematically affect normal modes of the guest molecule when it is on the side of the extracted inorganic matter compared to the case, when it is far from the edges of the silica construct. Also, since we deal with a large configurational space for silica, it may be questioned if geometry of relatively large extracts of this inorganic matter may contribute to systematic electronic correlations at sharp edges to modulate frequencies of a guest molecule. In other words, even though silicon oxide is a wide gap semiconductor, should we be aware of phenomena that are weak but analogous to those contributing to surface enhanced Raman? ${ }^{26}$ If weak but present, such phenomena would bias structural analysis. These questions become particularly critical since a recent publication on properties of silicon oxide, ${ }^{27}$ using methods of quantum mechanics/molecular mechanics, ${ }^{28}$ anticipates variation of force in dependence on distance from the silica surface (to account effects of contact between regions by classical and quantum mechanics) under finite and full periodic considerations applied for bulk $\mathrm{SiO}_{2}$ and an $\mathrm{OH}$ terminated $\mathrm{SiO}_{2}$ slab.

The purpose of this contribution is to address the question of possible influences of sites at silica interfaces on normal modes of a guest molecule. The guest molecules and their vibration normal modes (to use as spectral markers) were chosen to fulfil the following criteria: to serve as spectral markers to address the task, the normal modes should be relatively localized; that upon 
optimization of a guest molecule next to the surface the moieties involved in the vibration of interest would be located at an intermediate distance range above the surface, from 1.5 to $3 \AA$; that the vibrations of interest would not admix with the vibrations of the moieties specific to the inorganic interface; that it would be possible to conduct experimental studies to compare to theoretical predictions. To fulfil the criteria, we chose methanol as a small representative guest molecule positioned (in a model composite) next to the same type of silanol ${ }^{9}$ and under comparable geometries but at different locations - either next to an edge or in the middle of a flat region. We positioned methanols next to a double layer cluster extracted from a cristobalite system engineered in our previous MD simulation study. ${ }^{29}$ Next, we conducted DFT to explore the normal modes specific to the methyl moieties of the methanols and the electronic properties of silica. In our normal mode analysis we explore several schemes of isotope substitutions. This was necessary to understand how to learn and avoid possible admixing of the hydrogen bonded modes specific to the silica interface into the vibrations of the methanol molecules. Since the frequency difference attenuates cubic anharmonicity, ${ }^{30}$ detuning from the spectral region of $\mathrm{OH}$ vibrations attenuates contributions due to possible anharmonic interactions of the moieties involved in hydrogen bonded networks specific to the surface. To test the results of theoretical studies, we conducted FTIR experiment on the CD stretching modes of deuterated methanol when present as an impurity in water and in the presence of amorphous $\mathrm{SiO}_{2}$ nanoparticles. Additionally, we repeated such measurements after these particles were converted to crystalline cristobalite. The results reported here allow one to approach spectral predictions for molecular systems next to silica nanostructures and interfaces with a tested degree of rigor. Specifically, the reported results support our structural analysis of large polypeptides next to silicon oxide in large composite systems. Additionally, the 
methodological approach we employ here may be used to probe electronic properties and effects on vibrational properties of guest molecules in more challenging nanostructures.

\section{MATERIALS AND METHODS}

For the optical studies, we use $15 \mathrm{~nm}$ diameter amorphous silica nanoparticles synthesized by a modified Stöber method, ${ }^{31}$ and rehydrated cristobalite particles generated after thermal treatment for 4 hours at $1200{ }^{\circ} \mathrm{C}$ using a Furnace (RHF16/8 Carbolite Gero Ltd., Hope, UK). The size of the particles was measured by Dynamic Light Scattering (Malvern NanoS) and has been reported previously. ${ }^{9}$ The transformation to cristobalite was confirmed using a PANalytical X'Pert PRO, $\mathrm{X}$-ray powder diffractometer with $\mathrm{Cu} \mathrm{K \alpha}$ radiation with wavelength $1.54056 \AA$. Samples were scanned from $3^{\circ}$ to $50^{\circ}$ of $2 \theta$, accelerating voltage $45 \mathrm{kV}$, filament current $40 \mathrm{~mA}$ and scanning speed $0.02^{\circ} \mathrm{s}^{-1}$. X'Pert HighScore Plus (Version 2.0a) was used for pattern manipulation (baseline correcting and smoothing) and analysis. Figures $1 \mathrm{a}$ and $1 \mathrm{~b}$ demonstrate $\mathrm{X}$-ray diffraction scattering from a dry powder of silicon oxide amorphous nanoparticles and after high temperature conversion to cristobalite (includes peaks identified at 21.980, 31.459 and $36.0782 \theta$ (01-077-8627). FTIR studies were conducted with a Nicolet 6700 FTIR spectrometer, at a resolution of $1 \mathrm{~cm}^{-1}$. The ratio of $\mathrm{CD}_{3} \mathrm{OD}$ deuterated methanol (Sigma Aldrich 441384 ) to water was 1:30 by volume. Silica was added to give a dense but transparent paste. All samples were placed between two $2 \mathrm{~mm}$ thick calcium fluoride windows using a Teflon spacer of 100 microns.

For quantum mechanical studies we extracted a double layer of silicon oxide cluster, which contains 49 atoms of Silicon, from a slab of $\alpha$-cristobalite built and optimized in Molecular Dynamics simulation studies as reported earlier. ${ }^{29}$ The extracted cluster is $17.5 \AA \times 21 \AA$ in width 
and length, and about $3.2 \AA$ thick taking account of the distance between Si atoms in the two layers. The selected structure and dimensions allowed us to model two sites at the centers of each side to position two methanol guest molecules. In this contribution, we call these positions Centre- 1 and Centre-2 as shown in Figure 2. At these positions the guest methanol molecule would experience an environment comparable to that when next to a crystalline silicon oxide interface: as the radius of a Methyl group is $1.1 \AA$, the distance from the methyl group to the edges of the cluster would vary from 6.6 to $10 \AA$. To explore the effect of edges, we placed another two methanol molecules at the opposite sides of the cluster (we name these positions as Edge-1 and Edge-2), as shown in Figure 2. Special care was taken that the distance between the methanol molecules would be sufficient to avoid formation of hydrogen bonds between the methanol molecules. The methanol molecule at Centre-1 was positioned so that its $\mathrm{OH}$ moiety bridges two isolated $\mathrm{OH}$ groups at the silica interface. Comparatively, the $\mathrm{OH}$ group of the methanol at Edge-1 bridges a geminal and an isolated $\mathrm{OH}$ group at the silica interface. ${ }^{9}$ The Oxygen of the methanol at Edge- 2 coordinates one geminal and one isolated $\mathrm{OH}$ group of silica. Comparatively, the Oxygen of the methanol at Centre-2 coordinates two isolated $\mathrm{OH}$ groups at the silica interface.

We conducted quantum studies using Density Functional Theory (DFT) with the restricted B3LYP functional ${ }^{32,33}$ Comparing to performances of Hartree-Fock, Møller-Plesset, and local spin density approximation functionals, generalized gradient approximation calculations (GGAC) yield the best results to reproduce experimental bond lengths, angles, hydrogen bond interaction energies, heats of formation and ionization potentials. ${ }^{34}$ Among hybrid GGAC functionals, the relatively fast B3LYP is the most accurate for calculation of vibration frequencies. ${ }^{34,35}$ Since the molecular system under investigation in this contribution does not involve transition metals, for which B3LYP shows weak performance, ${ }^{36}$ the reported efficiency of the B3LYP hybrid functional 
was a major determining factor in our choice of functional for this study. We performed structural optimizations and normal mode calculations using the 6-31g* basis set within the Gaussian 09 program package. ${ }^{37}$ Structural optimization was conducted without any structural constraint methanol molecules were free to optimize their relative position in the locality of their initial placement. The basis set $6-31 \mathrm{~g}^{*}$ is the smallest we could use to receive optimal structure of the silica cluster with methanol molecules (system of 249 atoms) on a reasonable time scale (several months). However, we also conducted single point population analysis using the $6-31 \mathrm{G}(2 \mathrm{~d}, \mathrm{p})$ basis set to confirm that estimated electronic properties of the system would not suffer due to the level of theory: the data is provided in the Supporting Information. We employed the default setting of Gaussian 09 for the convergence criteria. Specifically, the convergence threshold for self consistent field integral accuracy was reached at $2.8 \times 10^{-9}$ Hartree, the threshold for maximum

force was $4.5 \times 10^{-4}$ Hartree/Bohr, for force root mean square was $3 \times 10^{-4}$ Hartree/Bohr, for maximum displacement was $1.8 \times 10^{-3} \mathrm{Bohr}$, and for displacement root mean square was $1.2 \times 10^{-3}$ Bohr. To explore the effects of position on the nature of the normal modes we calculated frequencies of methyl vibrations for methanol with Tritium, Deuterium and Hydrogen, or with three Deuterium atoms. The latter case being necessary to discuss the experimental results.

\section{RESULTS AND DISCUSSION}

A representative FTIR spectrum of the C-D stretching modes of deuterated methanol at impurity levels in water is shown in Figure 1c. The infrared absorption at $2079.9 \mathrm{~cm}^{-1}$ is specific to the mode when all three Deuterium atoms of the methyl moiety stretch together either towards the central Carbon atom or away. In the spectral region from 2120 to $2200 \mathrm{~cm}^{-1}$ there is a relatively 
weak Infrared absorption at about $2140 \mathrm{~cm}^{-1}$. Variances of such transitions have been considered to arise from overtones and combinations of methyl deformations. ${ }^{39}$ We conducted additional DFT anharmonic analysis on a small methanol cluster, see Supporting Information, and assigned such activities to combinations of $\mathrm{C}-\mathrm{O}$ stretchings (at about $1013 \mathrm{~cm}^{-1}$ ) and normal modes, where symmetric C-D umbrella bendings of a methyl group is mixed with C-O stretching (at about 1164 $\mathrm{cm}^{-1}$ ). Also, in this spectral region, one may expect contributions of overtones of D-C-D bendings, the fundamentals of which are at about $1110 \mathrm{~cm}^{-1}$.

Next, the resonance at $2233 \mathrm{~cm}^{-1}$ is of the mode where two Deuterium atoms orthogonal to the plane of the C-O-D backbone, stretch antisymmetrically and the Deuterium atom, which is in the plane of the C-O-D backbone is not moving. The vibration, where the two Deuterium atoms, that are orthogonal to the plane of the C-O-D backbone, stretch out symmetrically, while the Deuterium atom, which is in the plane of the C-O-D backbone, moves toward the Carbon atom, shows a resonance at $2253 \mathrm{~cm}^{-1}$. We may compare the observed responses in our studies with the spectral properties reported for nearly isolated deuterated methanol molecules in a solid argon matrix at $9 \mathrm{~K},{ }^{38}$ and for vibrations of $\mathrm{C}-\mathrm{D}$ moieties of chemisorbed $\mathrm{CD}_{3} \mathrm{OH}$ at the surface of silica at room temperature. ${ }^{39}$ In the former case, the corresponding resonances (Full Width at Half Maximum of $2 \mathrm{~cm}^{-1}$ ) have been observed and assigned as: $2078.3 \mathrm{~cm}^{-1}$ for $\mathrm{VCD}_{3}\left(\mathrm{~A}^{\prime}\right) \mathrm{CD}_{3}$ symmetric stretch, $2219.3 \mathrm{~cm}^{-1}$ for $\mathrm{vCD}_{3}\left(\mathrm{~A}^{\prime \prime}\right) \mathrm{CD}_{3}$ asymmetric stretch, and $2255.2 \mathrm{~cm}^{-1}$ for $v \mathrm{CD}_{3}\left(\mathrm{~A}^{\prime}\right) \mathrm{CD}_{3}$ asymmetric stretch. In the latter case, the spectral responses of chemisorbed $\mathrm{CD}_{3} \mathrm{OH}$ were complicated with additional spectral components at $2218,2273 \mathrm{~cm}^{-1}$, and background broadenings, which were due to the complexity of the normal modes and their combinations, which are expected to be present in various complex structural arrangements of deuterated methanols while under different geometries at binding sites at the silica interface. ${ }^{40}$ In 
our experiment, the responses are less complicated as we sample from solitary moieties, mainly. In Figures $1 \mathrm{~d}$ and $1 \mathrm{e}$ we show the difference spectra (in the presence of silica minus without). The results indicate that the effect of Silica particles on the C-D stretching modes of deuterated methanol results in a small but uniform (towards both lower and higher frequency directions) broadening.

To understand the experimental results better and gain a deeper insight into the physics and chemistry at the interface of silicon oxide we compared the experimental results to the spectral dispersions for methanol molecules modelled at different sites of the silica cluster prepared and optimized, as described in the materials and methods. Accordingly, in Figure 3a we present the spectra of the methyl modes when all three atoms next to each central Carbon are either Hydrogens, or Deuteriums, or Tritiums; a spectral signature of each of the four considered locations (sites) is shown in a color as assigned in Figure 2. Furthermore, for comparison in Figure 3b we show spectral signatures of hydrated methanol molecules in vacuum, where the three atoms next to each central Carbon are either Hydrogens, or Deuteriums, or Tritiums, also. The spectral simulations suggest that different locations of methanols next to silica do contribute to the anticipated spectral inhomogeneity. Of course, inhomogeneity is also expected when single methanol molecules or dimers would partition into various cavities of hydrogen bonded dynamic networks in an aqueous environment. However, comparing broadenings in different continuous environments is not the focus of this study. What is of a practical interest to us is to understand if there is a systematic trend in frequency shifts for the methanol C-D normal modes, when the molecule would be either next to an edge of the silicon oxide cluster or in the middle of a flat surface. From this perspective, first, it is helpful to check if the normal modes of the molecules at edges would demonstrate either a systematic blue shift or a systematic red shift. The theoretical anticipation for such (see red and 
orange lines in Figure 3a) indicates that the normal modes for methanols with Deuterium and Tritium atoms, while they are at different edges of the $\mathrm{SiO}_{2}$ cluster, demonstrate spectral responses of their normal modes that are shifted in opposite directions. The spectral properties at the edges suggest that there is not a systematic bias effect on the frequencies of the normal modes of the molecules at such sites. At the same time, it is important to stress here that the normal modes of the methanols in the centres of the flat regions of the cluster demonstrate weaker spectral dispersions.

To provide a deeper insight, in Figure 2 we present the Electrostatic Potential (the main contribution to generalized force ${ }^{27}$ at the interface) mapped on the Electron Densities visualized as the surface shown from inside, while taking views from the opposite sides of the cluster. We see that the character of electronic modulation at the surface is the same according to the spacing between Oxygens and Hydrogens of silanol groups, regardless of whether a site of interest is at the edge or in the middle of a relatively flat area. We do not observe any signatures of accumulated long range electronegative nor electropositive components toward the edges or anywhere else. There are no signatures of any long range electronic correlations to compete with local effects. This would change, for example, upon an inclusion of a noble metal cluster but this is beyond the scope of the current study. Even though there is a lack of systematic electron correlations, still, the calculated weaker dispersions of the normal modes of the methanol molecules in the centres of the flat regions suggest that material discontinuity of silica has only a weak effect on the normal modes of methanols.

Visualization of location and character of the Highest Occupied Molecular Orbital (HOMO) and Lowest Unoccupied Molecular Orbital (LUMO) provides further helpful diagnostics on the electronic properties in dependence on shape. In this respect, it is interesting to note that the 
HOMO of the system is localized mainly on the $\pi$-electrons of the Oxygens of the $\mathrm{OH}$ group of methanol and partially on the $\pi$-electrons of the Oxygens of the silanol group, to which the methanol is coordinated, see Figure 2. At the same time, the LUMO is associated with s states of silica on the other side of the cluster. These computational results also support the statement that the very local physics (within the radius of one $\mathrm{Si}-\mathrm{O}$ bond), according to geometry and electron hybridization, is likely to influence the nature of the normal modes and their frequencies.

It is important to notice that even if we calculate the normal modes for methanol where the methyl moiety includes naturally abundant Hydrogens, we do not rely on experimental results for such a system in the comparative discussion. The calculated spectra in Figure 3a provide the reason for us to use completely deuterated methanol at impurity levels in our experimental measurements. Specifically, exploring the spectral response of the $\mathrm{vCH}_{3}\left(\mathrm{~A}^{\prime}\right) \mathrm{CH}_{3}$ symmetric stretches of the methanol at Centre-1 we observe two responses (instead of the one expected), and both are 15 times scaled down. The complexity arises due to the fact that when using native methanol we should expect that via their $\mathrm{OH}$ groups the molecules would become involved in vibrations specific to the hydrogen bonded networks of silanol moieties and to the aqueous states next to the interface of the cluster. Since the frequencies of the $\mathrm{CH}_{3}$ group are close to the frequencies of the $\mathrm{OH}$ moieties at the interface, $\mathrm{CH}$ vibrations may couple to $\mathrm{OH}$ stretchings of the interface more effectively. As a result of slight admixing with the surface state, $\mathrm{CH}$ vibrations can gain in intensity (due to delocalizations) and may split into a subset of vibrations with different participations of the surface specific $\mathrm{OH}$ modes. To avoid this, in our experiment and in comparison with theoretical prediction we rely on the results for the systems where methanols carried Deuterium substitutions that provide a larger frequency separation from $\mathrm{OH}$ stretching and bending modes. 
It is also important to address the noticed effect of the harmonic coupling, which determines the expression of the described collective symmetric and antisymmetric vibrations. Of course, if complex in character and delocalized, the spectral signature of normal modes, which belong to the same subspace (like Amide I or C-H stretching modes), may hinder perception of possible tendencies due to the variances of physics if present at different sites at the surface. To address this we expressed the normal modes of the guest methanols (at different sites on the silica cluster) while reserving labelling with Tritium, Hydrogen and Deuterium for the methyl bonds oriented the most towards, away and intermediately toward the inorganic matter. We represent the results in Figure 3c. For comparison, in Figure 3d we express the normal modes of a single hydrated methanol while its three bonds are labelled with the three different isotopes while varying their relative positions. The results represented in Figure $3 \mathrm{c}$ show even more clearly (than using Figure 3a) that there are no systematic effects of positioning of a guest molecule either at the edges or in the flat regions of a cristobalite silicon oxide cluster of intermediate dimensions. We observe a uniform smearing in both, higher and lower frequency directions according to very local diversities and this is in general agreement with the experimental observations.

\section{CONCLUSIONS}

Silicon oxide plays a significant role in contemporary bioengineering. However, most practical initiatives are still somewhat intuitive. ${ }^{3-7}$ This is unfortunate because besides making research more effective we should note that some forms of silica are toxic and cancerogenic. ${ }^{41}$ The main difficulty in rigorous structural and functional predictions is in the fact that hybrid bio-inorganic composites (with silica) are large, heterogeneous and under different degrees of order and disorder. If using 
optical spectroscopy, structural analysis relies on calculations of normal modes. Attempting this in large composite systems requires bridging between classic and quantum simulations, when a small atomic set (of specific interest) is extracted from a classic simulation for further analysis using tools of quantum chemistry. To address this, in the article we explore (experimentally and theoretically) the possible spurious or systematic field effects of sharp edges (discontinuities) of silicon oxide on the normal modes of methanol molecules (as a proxy for more complicated molecules) associated with silica interfaces. When next to silicon oxide (either amorphous or cristobalite), we observe no systematic shifts in frequencies but a uniform broadening of calculated and detected optical transitions. Such broadening, however, is slightly narrower for molecules associated with flat regions compared to when the same molecules are next to edges. The results of the studies remove possible concerns about the effect of structural discontinuities of the inorganic matter as long as the chemical variances, such as deprotonated $\equiv \mathrm{Si}-\mathrm{O}$ - groups and/or proximal ions are accounted for explicitly. Indeed, classic simulation can provide information on such chemical variances. ${ }^{10,29}$ The adopted approach may be extended to more complex metallic or metallized (by doping) oxide systems of reduced dimensions and complex geometry.

\section{AUTHOR INFORMATION}

\section{Corresponding Author}

*Carole C. Perry. Email: carole.perry@ntu.ac.uk.

\section{Author Contributions}

The manuscript was written through contributions of all authors.

\section{Notes}


The authors declare no competing financial interest.

\section{Supporting Information}

HOMO, LUMO, Electrostatic potentials mapped onto electronic densities and Total Densities of States of the considered silica cluster with guest methanols using 6-31G(d) and 6-31G(2d,p) basis sets. Infrared absorptions of fundamental normal modes, combinations and overtones of methanol cluster by DFT anharmonic analysis.

\section{ACKNOWLEDGMENT}

Funding from AFOSR FA9550-16-1-0213 is gratefully acknowledged by the authors. 


\section{FIGURE CAPTIONS}

Figure 1. X-Ray diffraction from a dry powder of amorphous silicon oxide nanoparticles as synthesized (a) and after high temperature conversion to cristobalite (b). FTIR spectrum of a sample, where $\mathrm{CD}_{3} \mathrm{OD}$ methanol is dissolved in water at a ratio $1: 30 \mathrm{v} / \mathrm{v}(\mathrm{c})$. For the same deuterated methanol to water ratio we prepared samples in the presence of amorphous silica and cristobalite particles and we measured two FTIR spectra for such sample. (d) and (e): spectrum in the presence of silica minus the spectrum without silica (as in (c)) for amorphous and cristobalite cases, respectively.

Figure 2. Left: graphic representations for DFT optimized silica cristobalite cluster with associated methanol molecules at the two central and the two edge sites, as indicated. Top and bottom panels represent the views of the cluster from its side and when its lower front edge (as in the top image) is slightly uplifted. The labelling colours correspond to the colours of calculated spectra, as shown in Figures 3(a) and 3(c). The provided labelling for isotope substitution corresponds to the calculated spectra, as shown in Figure 3(c). Middle: graphic representation of HOMO and LUMO. Right: Electrostatic Potential mapped on the Electron Densities visualized as surfaces from inside of the system using opposite points of view.

Figure 3. (a): black and grey, red and orange lines show calculated spectra for four methanol molecules placed at two central sites and two sites at the edges of a $\mathrm{SiO}_{2}$ cluster (graphical presentation is given in Figure 2), respectively, while the methyl moieties are isotopically labelled either with three Tritiums, or with three Deuteriums, or with Hydrogens. The colour of the lines reflects the location of the sites. The panel compares detected FTIR with the calculated spectra for $\mathrm{HO}-\mathrm{CD}_{3}$. Figure 3. (b): black, blue and magenta lines show calculated spectra for hydrated 
methanol in vacuum when its methyl is isotopically labelled either with three Tritiums, or three Deuteriums, or three Hydrogens. (c): black and grey lines represent calculated spectra for two methyl molecules placed at two central sites, and red and orange lines show the calculated spectra for two molecules at the edges of the $\mathrm{SiO}_{2}$ cluster, while each methyl would have a Tritium, a Deuterium and a Hydrogen. In each case, the isotopes were placed such that a Tritium and a Hydrogen would point toward and away from the surface of the silica cluster, respectively. (d): calculated spectra for hydrated methanol in vacuum when its methyl is isotopically labelled with a Tritium, a Deuterium and a Hydrogen atom. We use different colours for spectra here to show that under different placements of isotopes there is no significant variance in calculated frequencies. The frequency scaling factor is 0.955 . 


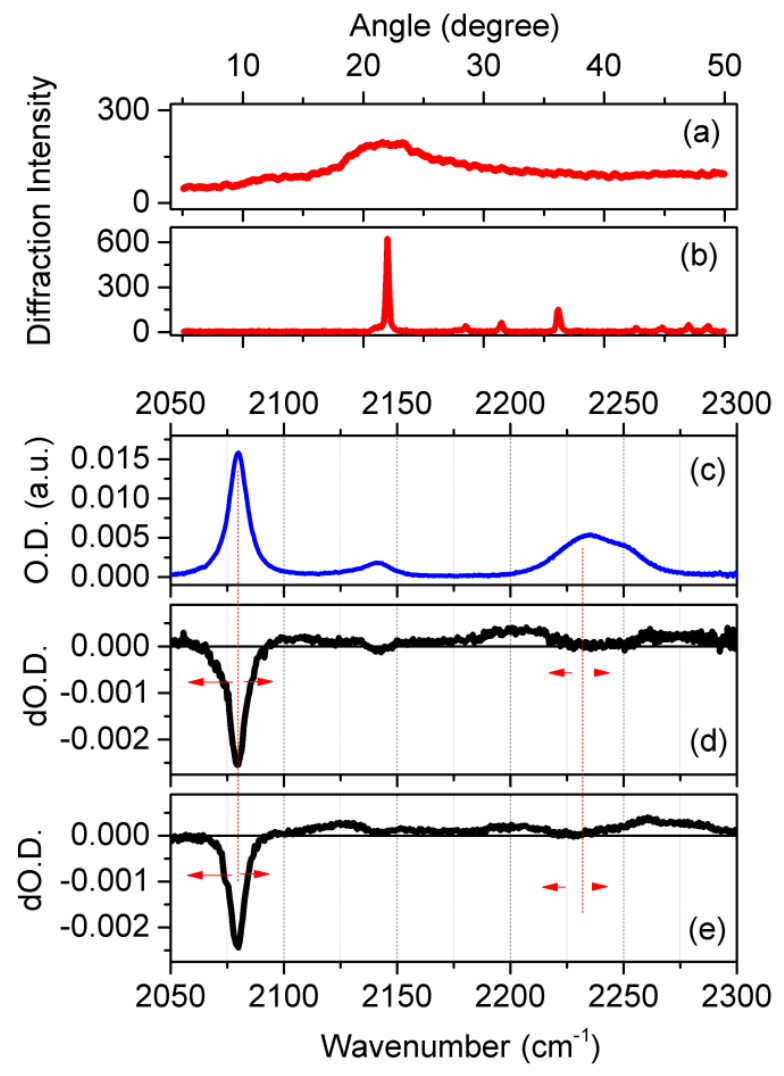

Figure 1. 

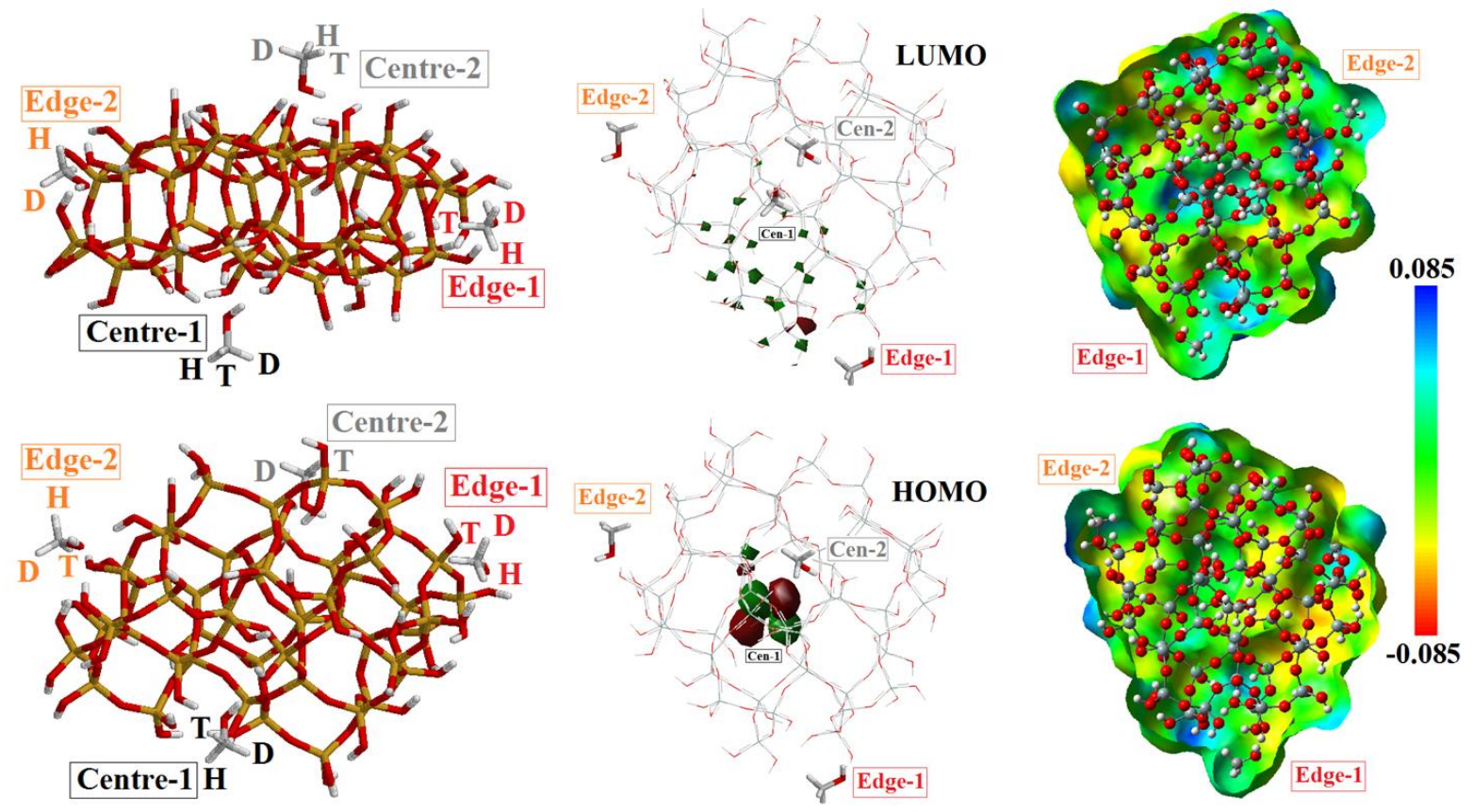

Figure 2. 


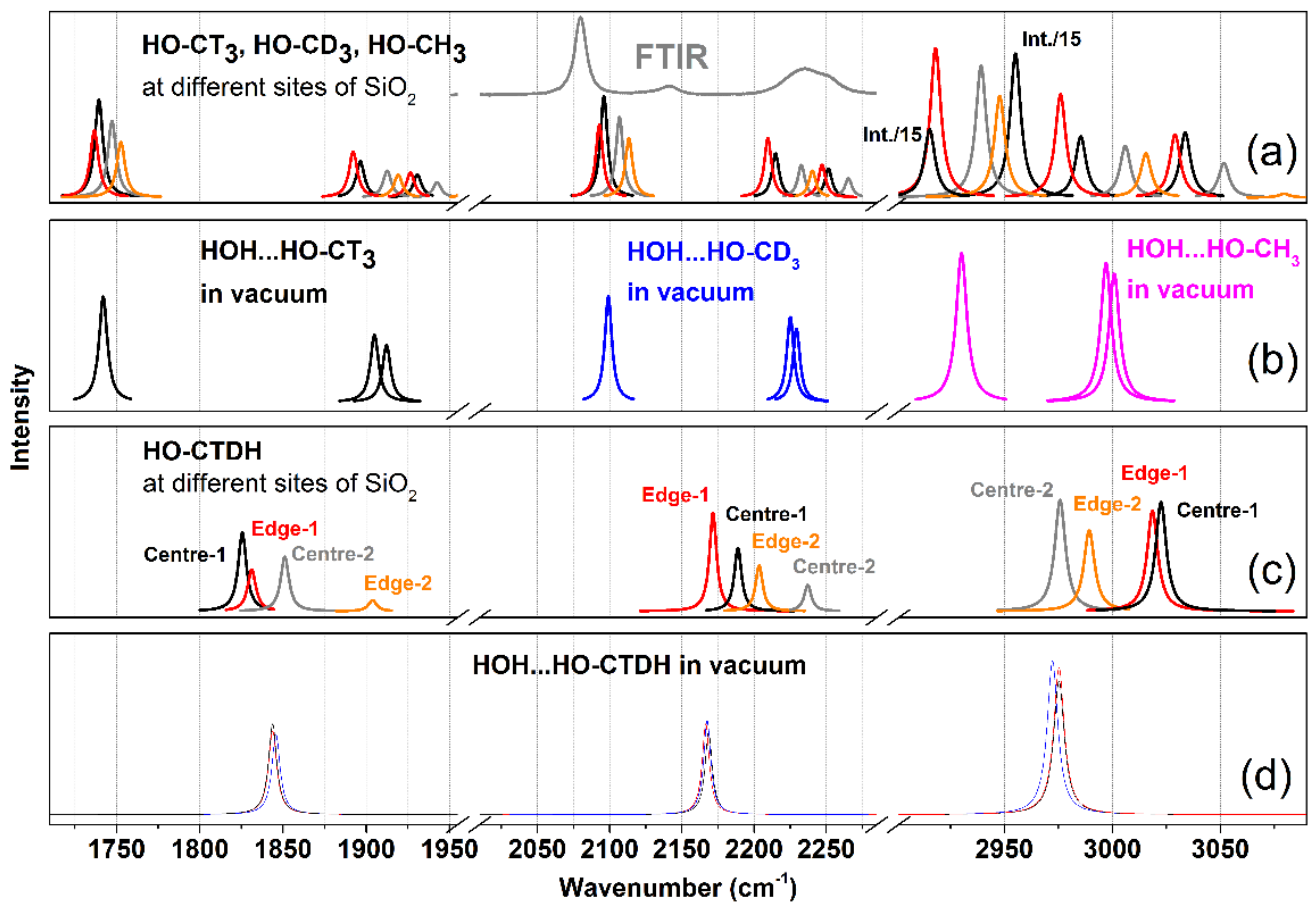

Figure 3 


\section{REFERENCES}

(1) Dana, J. D.; Dana, E. S. Silica Minerals. The System of Mineralogy of James Dwight Dana and Edward Salisbury Dana; Vol.3, John Wiley \& Sons: New York, 1962.

(2) Iler, K. The Chemistry of Silica: Solubility, Polymerization, Colloid and Surface Properties and Biochemistry; Wiley-Interscience: New York, 1979.

(3) Giraldo, L. F.; López, B. L.; Pérez, L.; Urrego, S.; Sierra, L.; Mesa, M. Mesoporous Silica Applications; Macromol. Symp. 2007, 258, 129-141.

(4) Pagliaro, M. Silica-based Materials for Advanced Chemical Applications; RSC Publishing: Cambridge, U.K. 2009.

(5) Qian, K. K.; Bogner, R. H. Application of Mesoporous Silicon Dioxide and Silicate in Oral Amorphous Drug Delivery Systems. J. Pharm. Sci. 2012, 101, 444-463.

(6) Pacchioni, G.; Skuja, L.; Griscom, D. L. Defects in $\mathrm{SiO}_{2}$ and Related Dielectrics: Science and Technology; NATO Science Series, Series II: Mathematical And Physical Chemistry; Dordrecht: Kluwer, 2000.

(7) Devine, R. A. B.; Duraud, J.-P.; Dooryhee, E. Structure and Imperfections in Amorphous and Crystalline Silicon Dioxide; New York: Wiley, 2000.

(8) Waldrop, M. M. The Chips are down for Moore's Law. Nature 2016, 530, 145-147.

(9) Rimola, A; Costa, D.; Sodupe, M.; Lambert, J.-F.; Ugliengo, P. Silica Surface Features and their Role in the Adsorption of Biomolecules: Computational Modeling and Experiments. Chem. Rev. 2013, 113, 4216-4313. 
(10) Patwardhan, S. V.; Emami, F. S.; Berry, R. J.; Jones, S. E.; Naik, R. R.; Deschaume, O.; Heinz, H.; Perry, C. C. Chemistry of Aqueous Silica Nanoparticle Surfaces and the Mechanism of Selective Peptide Adsorption. J. Am. Chem. Soc. 2012, 134, 6244-6256.

(11) Szymanski, M. A.; Shluger, A. L.; Stoneham, A. M. Role of Disorder in Incorporation Energies of Oxygen Atoms in Amorphous Silica. Phys. Rev. B 2001, 63, 224207.

(12) Donadio, D.; Bernasconi, M.; Boero, M. Ab Initio Simulations of Photoinduced Interconversions of Oxygen Deficient Centers in Amorphous Silica. Phys. Rev. Lett. 2001, 87, 195504.

(13) Tielens, F.; Gervais, C.; Lambert, J. F.; Mauri, F.; Costa, D. Ab Initio Study of the Hydroxylated Surface of Amorphous Silica: A Representative Model. Chem. Mater. 2008, $20,3336-3344$.

(14) Rimola, A; Ugliengo, P; Sodupe, M. Strained Ring Motif at Silica Surfaces: a Quantum Mechanical Study of their Reactivity towards Protic Molecules. Comp. Theor. Chem. 2015, $1074,168-177$.

(15) Sushko, P. V.; Mukhopadhyay, S.; Mysovsky, A. S.; Sulimov, V. B.; Taga, A.; Shluger, A. L. Structure and Properties of Defects in Amorphous Silica: New Insights from Embedded Cluster Calculations. J. Phys.: Condens. Matter 2005, 17, S2115-S2140.

(16) Diez, R.P.; Amalvy, J. I. A Density Functional Study of the Adsorption of Pyridine 2vinylpyridine, and 4-vinylpyridine onto a Silica Surface. J. Mol. Struct. (Theochem.) 2003, 634, 187-193. 
(17) Singha, K.; Chandraa, B.; Rhymanb, L.; Ramasami, P. Effective Adsorption of Pyridine (Py)—onto Mesoporous Silica derived from De-oiled Mustard Cake (DOMC): Experimental and Theoretical study. J. Env. Chem. Eng. 2016, 4, 1383-1392.

(18) Lomenech, C.; Bery, G.; Costa, D.; Stievano, L.; Lambert, J. F. Theoretical and Experimental Study of the Adsorption of Neutral Glycine on Silica from the Gas Phase. Chem. Phys. Chem. 2005, 6, 1061-1070.

(19) Abbasi, A.; Nadimi, E.; Plänitz, P.; Radehaus, C. Density Functional Study of the Adsorption of Aspirin on the Hydroxylated (001) Alpha-Quartz Surface. Surf. Sci. 2009, 603, 2502-2506.

(20) Piane, M. D.; Vaccari, S.; Corno, M.; Ugliengo, P. Silica-Based Materials as Drug Adsorbents: First Principle Investigation on the Role of Water Microsolvation on Ibuprofen Adsorption. J. Phys. Chem. A 2014, 118, 5801-5807.

(21) Vreven, T.; Mennucci, B.; da Silva, C. O.; Morokuma, K.; Tomasi, J. The ONIOM-PCM Method: Combining the Hybrid Molecular Orbital Method and the Polarizable Continuum Model for Solvation. Application to the Geometry and Properties of a Merocyanine in Solution. J. Chem. Phys. 2001, 115, 62-72.

(22) Pedone, A.; Prampolini, G.; Monti, S.; Barone, V. Absorption and Emission Spectra of Fluorescent Silica Nanoparticles from TD-DFT/MM/PCM Calculations. Phys. Chem. Chem. Phys. 2001, 13, 16689-16697. 
(23) Ugliengo, P.; Sodupe, M.; Musso, F.; Bush, I. J.; Orlando, R.; Dovesi, R. Realistic Models of Hydroxylated Amorphous Silica Surfaces and MCM-41 Mesoporous Material Simulated by Large-Scale Periodic B3LYP Calculations. Adv. Mater. 2008, 20, 4579-4583.

(24) Puddu, V.; Perry, C. C. Interactions at the Silica-Peptide Interface: The Influence of Particle Size and Surface Functionality. Langmuir 2014, 30, 227-233.

(25) Oren, E. E.; Notman, R.; Kim, I. W.; Evans, J. S.; Walsh, T. R.; Samudrala, R.; Tamerler, C.; Sarikaya, M. Probing the Molecular Mechanisms of Quartz-Binding Peptides. Langmuir 2010, 26, 11003-11009.

(26) Ford, G. W.; Weber, W. H. Electromagnetic interactions of molecules with metal surfaces. Phys. Rep. 1984, 113, 195-287.

(27) Peguiron, A.; Colombi Ciacchi, L.; De Vita, A.; Kermode, J. R.; Moras, G. Accuracy of Buffered-force QM/MM Simulations of Silica. J. Chem. Phys. 2015, 142, 064116.

(28) Warshel, A.; Levitt, M. Theoretical Studies of Enzymic Reactions: Dielectric, Electrostatic and Steric Stabilization of the Carbonium Ion in the Reaction of Lysozyme. J. Mol. Biol. 1976, 103, 227-249.

(29) Emami, F.S.; Puddu, V.; Berry, R. J.; Varshney, V. S.; Patwardhan, V.; Perry, C. C.; Heinz, H. Force Field and a Surface Model Database for Silica to Simulate Interfacial Properties in Atomic Resolution. Chem. Mat. 2014, 26, 2647-2658.

(30) Califano, S. Vibrational states. Wiley, 1976.

(31) Stober, W.; Fink, A. Controlled Growth of Monodispersed Silica Spheres in the Micron Size Range. J. Coll. Int. Sci. 1968, 26, 62-69. 
(32) Becke, A. D. Density-functional Exchange-energy Approximation with Correct Asymptotic Behavior. Phys. Rev. A 1988, 38, 3098-3100.

(33) Lee, C.; Yang, W.; Parr, R. G. Development of the Colle-Salvetti Correlation-energy Formula into a Functional of the Electron Density. Phys. Rev. B 1988, 37, 785-789.

(34) Riley, K. E.; Op’t Holt, B. T.; Merz Jr., K. M. Critical Assessment of the Performance of Density Functional Methods for Several Atomic and Molecular Properties. J. Chem. Theory Comput. 2007, 3, 407-433.

(35) El-Azhary, A. A.; Suter, H. U. Comparison Between Optimized Geometries and Vibrational Frequencies Calculated by the DFT Methods. J. Phys. Chem. 1996, 100, 1505615063.

(36) Cramer, C. J.; Truhlar, D. G. Density Functional Theory for Transition Metals and Transition Metal Chemistry. Phys. Chem. Chem. Phys. 2009, 11, 10757-10816.

(37) Frisch, M. J.; Trucks, G. W.; Schlegel, H. B.; Scuseria, G. E.; Robb, M. A.; Cheeseman, J. R.; Scalmani, G.; Barone, V.; Mennucci, B.; Petersson, G. A. et al. Gaussian 09, revision e.01, Gaussian, Inc., Wallingford CT, 2016.

(38) Han, S. W.; Kim, K. Infrared Matrix Isolation Study of Acetone and Methanol in Solid Argon. J. Phys. Chem. 1996, 100, 17124-17132.

(39) Morrow, B. A. Infra-red Studies of Reactions on Oxide Surfaces. Part 2. Methanol on Silica. J. Chem. Soc. Faraday Trans. I, 1974, 70, 1527-1545. 
(40) Pelmenschikov, A. G.; Morosi, G.; Gamba, A. Adsorption of Water and Methanol on Silica Hydroxyls: Ab Initio Energy and Frequency Calculations. J. Phys. Chem. A 1997, 101, 11781187.

(41) Leung, C. C.; Yu, T. S.; Chen, W. Silicosis. The Lancet 2012, 379, 2008-2018. 


\section{TOC Graphic}

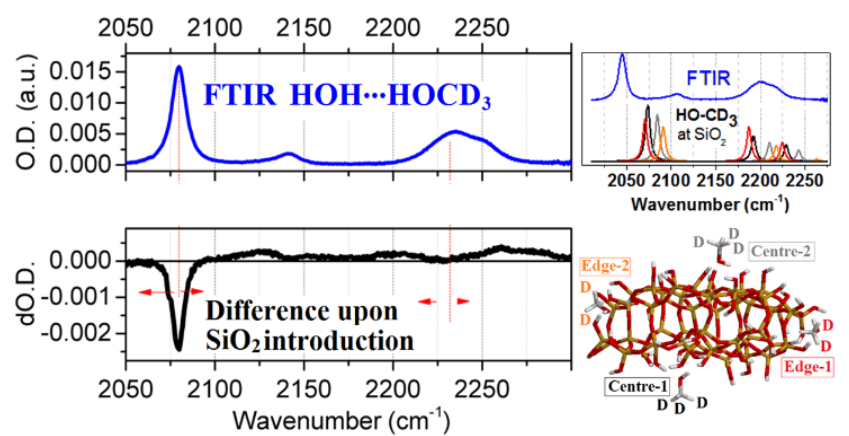

